\title{
Potential-Modulation Spectroscopy at Solid/Liquid and Liquid/Liquid Interfaces
}

\author{
Hirohisa Nagatani and Takamasa SAgara ${ }^{\dagger}$ \\ Department of Applied Chemistry, Faculty of Engineering, Nagasaki University, \\ Bunkyo 1-14, Nagasaki 852-8521, Japan
}

\begin{abstract}
Potential-modulation spectroelectrochemical methods at solid/liquid and liquid/liquid interfaces are reviewed. After a brief summary of the basic features and advantages of the methods, practical applications of potential-modulation spectroscopy are demonstrated using our recent studies of solid/liquid and liquid/liquid interfaces, including reflection measurements for a redox protein on a modified gold electrode and fluorescence measurements for various dyes at a polarized water/1,2-dichloroethane interface. For both interfaces, the use of linearly polarized incident light enabled an estimation of the molecular orientation. The use of a potential-modulated transmission-absorption measurement for an optically transparent electrode with immobilized metal nanoparticles is also described. The ability of potential-modulated fluorescence spectroscopy to clearly elucidate the charge transfer and adsorption mechanisms at liquid/liquid interfaces is highlighted.
\end{abstract}

(Received April 9, 2007; Accepted July 3, 2007; Published September 10, 2007)

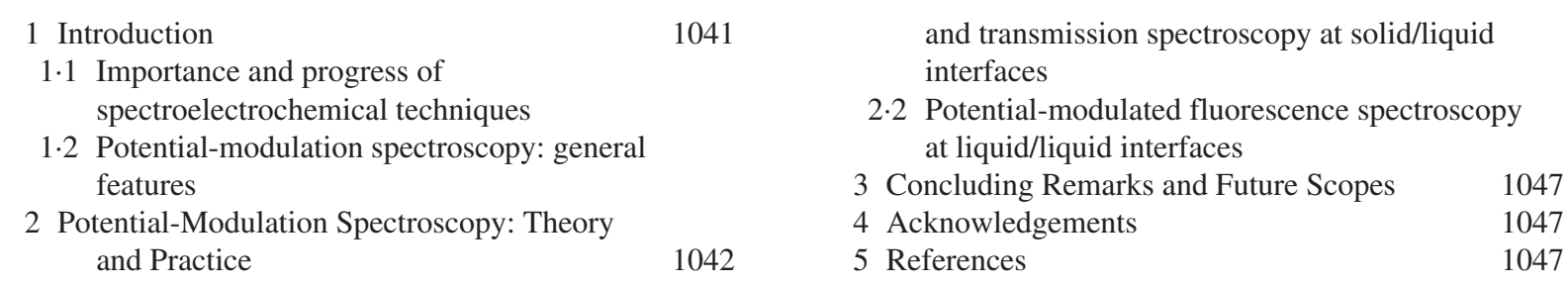

$2 \cdot 1$ Potential-modulated UV-visible reflectance

\section{Introduction}

1.1 Importance and progress of spectroelectrochemical techniques

In the burgeoning field of interfacial science, in situ application of spectroscopic analysis is indispensable for understanding of the structure of the interface and the behavior of molecules therein. Spectroelectrochemical methods refer to the combined, simultaneous use of spectroscopic analytical tools and the electrochemical methods for the characterization of electrified interfaces. ${ }^{1}$ The irradiation of probe light to the interface and detection of the spectroscopic response under potential-control are the fundamental procedures of spectroelectrochemical measurements, except for the analysis of light emission from the interface without incident light excitation. As long as the incident probe light reaches the interface and the signal light comes back to the detector, we can use a number of types of spectroscopic techniques. The wavelength of the light ranges from X-ray to microwave, and even radiowave for in situ electrochemical NMR. ${ }^{2}$ Recently, methods using neutron radiation from an atomic

$\dagger$ To whom correspondence should be addressed.

E-mail: sagara@nagasaki-u.ac.jp reactor have also joined the family of spectroelectrochemical methods. $^{3} \quad$ The optical process as the origin of the spectroelectrochemical signal includes absorption, reflection, scattering, emission, non-linear optical processes and others.

After Shikata pointed out the importance of the combined use of polarographic and spectral methods ${ }^{4}$ the first bloom of spectroelectrochemical studies was recorded in the 1960's with in situ reflection measurements in the UV-visible wavelength region, ${ }^{5,6}$ followed by its extension to IR. ${ }^{7}$ In line with rapid developments in light sources, detectors, monochromators, optical elements, electronic hard- and softwares as well as cell design, the multiplex use of spectroelectrochemical techniques is now the key to reaching a conclusion about what is really occurring at the electrode/solution and liquid/liquid interfaces.

Substantial needs for the development of spectroelectrochemical methods have been continuously invoked. As the view from analytical science, not only exploration of the new spectroscopic methods, or their introduction to electrochemical research, but also a revisit to classical methods with an aim at improvement and utility widening should greatly contribute to our gaining a deeper insight into a variety of electrochemical processes, and to the development of sophisticated analytical methods. Among the spectroelectrochemical methods, those available with UV-visible incidence and/or emitted light are the 
most convenient for applications, because the transparency for both the probe and the signal light through the medium greatly simplifies the experimental setup, together with the removal of restrictions in the geometrical setting of the interface. Fluorescence spectral measurements are quite sensitive for an electrified interface, where a strong quenching environment is absent, because the signal has no background, in principle. Vibration spectral measurements, such as IR reflection, Raman scattering, and sum frequency generation spectroscopies, are also important to see the molecular structure details. When applying these techniques, modulation methods provide a high sensitivity and capability of tracking the dynamic behavior of molecules.

In this review, we focus on recent progress and applications of potential-modulation spectroelectrochemical methods at solid/liquid and liquid/liquid interfaces. For both interfaces, the advantages of the use of potential-modulation methods are highlighted using our recent experimental results obtained in the $\mathrm{UV}$-visible wavelength region.

\subsection{Potential-modulation spectroscopy: general features}

When our interests are in the detection of minimal changes of the structures and the states of the molecules at an electrified interface, a dc measurement of a spectroelectrochemical signal is frequently suffered with both a lack of sensitivity and stability and an inaccessibility to interfacial dynamics. It sometimes appears to be challenging to minimize the drift of the spectrophotometer due to the probe light intensity fluctuation, the influence of mechanical vibration, and electromagnetic noise. On the other hand, an ac measurement of a spectroelectrochemical signal with a potential-modulation perturbation enables us to detect only the ac change induced by the modulation. It can exclude from the signal to be obtained the superimposed dc component, ac components at different frequencies, and random noises. Therefore, we can achieve higher sensitivity, stability, and $S / N$ ratio. For example, when we should detect by a dc measurement the change of one part per thousand added on an unchanged thousand-level dc signal, we should offset the thousand-level of the dc part before amplification, or increase the full detection range by 3 orders of magnitude. In an ac measurement, in contrast, we can directly see an ac change of one part per thousand.

However, in order for the ac measurements to be conducted meaningfully, the spectroelectrochemical signal should oscillate in harmony with the ac potential-modulation. Therefore, the origin of the signal should be a chemically reversible process. In addition, we can gain access to the difference of the spectra between two states, but not to the absolute spectrum at a given potential.

In spite of the above-mentioned restriction in applying an ac method, the detection of a spectroelectrochemical signal as a response to only ac potential-modulation appears to be a powerful tool in spectroelectrochemistry. The advantages include not only the sensitive detection of a very small change of the spectroscopic signal exclusively induced by the modulation, but also the capability of tracking the kinetics. The phase shift of the output spectroscopic ac signal with respect to the input ac modulation directly reflects how fast the system follows the potential change, as discussed later.

In this review, we deal with only the modulation of the potential, while a number of other parameters can also be the modulation input, for example, wavelength, ${ }^{8}$ intensity and polarization of the probe light, photo-excitation, ${ }^{9}$ and current. As the origin of the ac spectroscopic signal, even if we limit the scope of the UV-visible wavelength region, a variety of optical processes can be used, including internal and external reflection, transmission, fluorescence, Rayleigh and Raman scattering, and non-linear optical processes.

In Section 2, a description of the potential-modulated UVvisible reflectance and transmission-absorption spectroscopies at solid/liquid interfaces is followed by that of potentialmodulated fluorescence spectroscopy at liquid/liquid interfaces.

\section{Potential-Modulation Spectroscopy: Theory and Practice}

2.1 Potential-modulated UV-visible reflectance and transmission spectroscopy at solid/liquid interfaces

The title reflection method is referred to as electroreflectance (ER) spectroscopy and the transmission method to potentialmodulated transmission-absorption (PMTA) spectroscopy. Because a number of excellent reviews for ER spectroscopy have already been published with rather comprehensive contents, ${ }^{1,6,10}$ we give herein a brief summary of the method, and then highlight our recent application for a redox protein. For PMTA spectroscopy, our recent application for a gold nanoparticle (Au-NP)-immobilized indium-tin oxide (ITO) electrode is used as an example.

\section{2·1·1 Electroreflectance (ER) spectroscopy}

Monochromatic probe light with a steady state intensity, $I_{0}$, is irradiated to a mirror-like flat surface of an electrode, the potential of which is ac modulated as described by the following equation using a complex representation:

$$
E=E_{\mathrm{dc}}+E_{\mathrm{ac}}=E_{\mathrm{dc}}+\Delta E_{\mathrm{ac}} \exp (j \omega t)
$$

where $E_{\mathrm{dc}}$ is the dc potential, $E_{\mathrm{ac}}$ is the ac potential, $\Delta E_{\mathrm{ac}}$ is the zero-to-peak amplitude of $E_{\mathrm{ac}}, j$ is an imaginary number, $\omega=$ $2 \pi f$ is the angular frequency ( $f$ is the frequency in a unit of $\mathrm{Hz}$ ), and $t$ is the time. When the value of $\Delta E_{\text {ac }}$ is small enough to give a linear response, the reflected light intensity, $I_{\mathrm{r}}$, is given as

$$
I_{\mathrm{r}}=I_{0} R=I_{0}\left[R_{\mathrm{dc}}+R_{\mathrm{ac}}\right]=I_{0}\left[R_{\mathrm{dc}}+\Delta R_{\mathrm{ac}} \exp \{j(\omega t+\phi)\}\right],
$$

where $R_{\mathrm{dc}}$ and $R_{\mathrm{ac}}$ are the $\mathrm{dc}$ and ac reflectance, respectively, $\Delta R_{\mathrm{ac}}$ is the amplitude of $R_{\mathrm{ac}}$, and $\phi$ is the phase of $R_{\mathrm{ac}}$ with respect to $E_{\mathrm{ac}}$. The term $\phi$ represents how fast the electrode surface process can follow $E_{\mathrm{ac}}{ }^{10}$ Phase-sensitive detection of the $I_{0} R_{\mathrm{ac}}$ (the ac part of $I_{\mathrm{r}}$ at an angular frequency of $\omega$ ) by a lock-in amplifier with simultaneous monitoring $I_{0} R_{\mathrm{dc}}$ as timeaveraged $R$ allows us to record the ER signal, which is defined as $R_{\mathrm{ac}} / R_{\mathrm{dc}}$ and designated as $(\Delta R / R)_{\mathrm{ER}}$. The ER signal possesses both real and imaginary parts. The former is its in-phase component to $E_{\mathrm{ac}}$, and the latter is the $90^{\circ}$ out-of-phase component. In another form, the relationship is expressed as $\cot \phi=-\operatorname{Re}\left[(\Delta R / R)_{\mathrm{ER}}\right] / \operatorname{Im}\left[(\Delta R / R)_{\mathrm{ER}}\right]$. A simplified scheme of the ER measurement setup is shown in Fig. 1. An ER spectrum, a plot of the ER signal as a function of the incident light wavelength $(\lambda)$, represents the reflection spectrum at positive potential, from which that at negative potential was subtracted.

Any potential-responsible, chemically reversible process at an electrode surface gives rise to a non-zero ER signal as long as it is accompanied by a change in the optical properties of the interface, regardless of whether it takes place at the surface, in the solution proximity of the surface, or both. Such processes include adsorption-desorption and an orientation change of a chromophore, a change of the interaction between a double-layer static electric field and the transition dipole of a chromophore (i.e. so-called electrochemical Stark effect), ${ }^{11}$ and even a change 


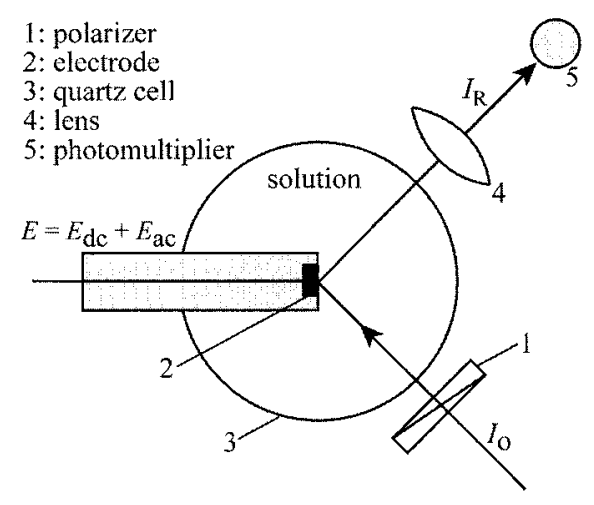

Fig. 1 Scheme of the setup for the ER measurement.

of the density of the surface free electrons of a metal electrode. In addition to the above-listed non-faradaic processes, redox reactions give rise to ER signals, if the absorption spectrum of the reduced form is different from that of the oxidized form. In this case, the ER spectrum actually corresponds in many cases to the difference absorption spectrum between two different oxidation states, by the use of which a discussion about the state of redox-active molecules at the interface is allowed.

As noted in $\mathbf{1 \cdot 2}$, the analysis of real and imaginary-part ER signals allows one to elucidate the kinetics and reaction mechanism and to discriminate of more-than-one reactions that take place simultaneously. This is also the case for PMTA at transparent electrode/solution interfaces as well as for potentialmodulated reflectance and fluorescence measurements at liquid/liquid interfaces; the latter is explicitly demonstrated in 2.2. It is worthwhile to note here two essential points in the analysis using both real and imaginary parts:

(1) When one observes a single-component electrochemical process by potential-modulation methods at a given $E_{\mathrm{dc}}$, the real and imaginary parts should give the same spectrum structure at any frequencies, yet their sign and intensity are not necessarily the same. This is an important diagnostic criterion in analytical interfacial electrochemisty.

(2) Being similar to impedance spectroscopy, one can represent real and imaginary-part signals as a function of the modulation frequency on a complex plane. This data can be analyzed on the basis of an interfacial equivalent circuit including solution resistance and double-layer capacitance (therefore, an ohmic drop correction can be made while performing data analysis after measurements, if necessary). When the ER signal is due to a redox reaction, we can obtain a heterogeneous electron-transfer rate constant. ${ }^{10}$ When the potential-modulated fluorescence signal is due to adsorptiondesorption at a liquid/liquid interface, related kinetic parameters are obtained (see $\mathbf{2} \cdot \mathbf{2}$ ). In general, a more sluggish process gives rise to a greater imaginary part relative to the real part at lower frequencies.

\subsubsection{ER spectral measurement as exemplified by redox of a heme protein}

A typical example for the redox ER signal of cytochrome $c$ (Cyt-c), a mono-heme protein, immobilized on an polycrystalline $\mathrm{Au}$ electrode modified with a self-assembled monolayer (SAM) of 11-mercapto-undecanoic acid (MUA), is shown in Fig. 2 as an ER spectrum. ${ }^{12}$ The spectrum represents the absorption spectrum of Cyt-c with the ferro-heme, from which that of the ferri-heme is subtracted. In cases like this, where chromophores are incorporated in a transparent medium dispersively without direct contact with the electrode surface,

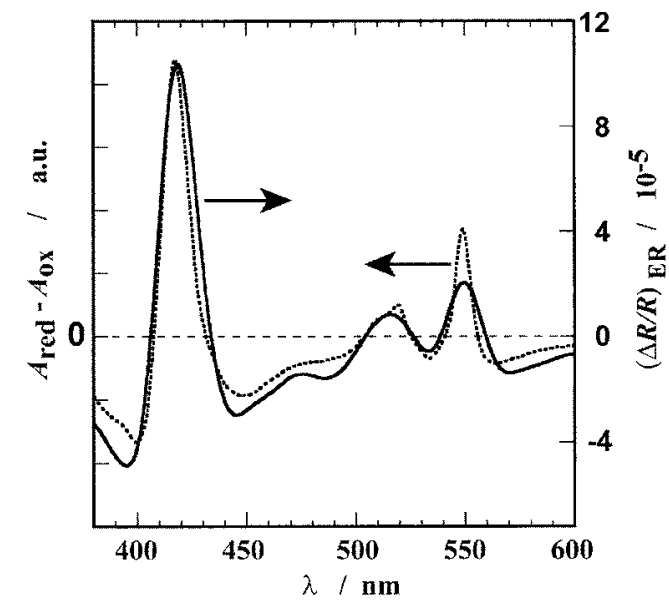

Fig. 2 Typical ER spectrum for a MUA-SAM modified Au electrode on which Cyt-c was immobilized. The electrolyte solution was $3.0 \times 10^{-2} \mathrm{~mol} \mathrm{dm}^{-3}$ phosphate buffer $(\mathrm{pH} \mathrm{7.0)}$. The ER spectrum (solid line) represents the real part measured at $E_{\mathrm{dc}}=E^{0^{\prime}}$ (formal potential), $f=14 \mathrm{~Hz}$, and $\Delta E_{\mathrm{ac}}=70 \mathrm{mV}_{\text {rms }}$ with p-polarized incident light with an incident angle $(\theta)$ of $45^{\circ}$. The dotted line is the solution absorption spectrum of ferrous Cyt-c from which that of ferric Cyt-c was subtracted. Reproduced with permission from Ref. 12. Copyright 2006 American Chemical Society.

the ER spectrum usually corresponds to the difference absorption spectrum. However, in the case that a dye layer covers the electrode surface by direct contact, the ER spectrum is far different from the difference absorption spectrum. In such a case, interpretation of the ER spectrum should be made based on an interfacial model with optical theories. ${ }^{13}$ Note that the amount of immobilized Cyt-c for Fig. 2 is $5 \times 10^{12}$ molecules $\mathrm{cm}^{-2}$, demonstrating the high sensitivity of the ER spectral measurement.

It has been predicted for Cyt-c immobilized on this SAM by many dc spectroscopic measurements ${ }^{14}$ and simulations ${ }^{15}$ that the molecules are uniformly oriented, so that the heme crevice of Cyt-c is totally facing the SAM but not the solution phase, while some reports have rebuted this model..$^{16}$ It is desirable to experimentally estimate the orientation of the heme by multiple spectroscopic methods. Because the light absorption of an orientated chromophore depends on the polarization of the probe light, an ER measurement with polarized incident light should be well-suited as one of the methods to monitor the orientation of molecules.

Previously, we developed a method to obtain the orientation of dyes adsorbed on electrode surfaces by using p- and s-polarized incident light in ER spectral measurements. ${ }^{17}$ The ratio of the ER signal with p-polarized light against that with s-polarized light (p/s-ratio) was described as a function of the incident angle of the probe light $(\theta)$ with respect to the surface normal when the chromophore to be probed possesses a single transition dipole. This formulation has been experimentally verified for a monolayer of viologen thiol (VT) on a Au electrode. ${ }^{17}$ However, the absorption of heme (iron protoporphyrin IX) is due to doubly degenerated transitions. When degeneration occurs for two orthogonal dipoles with an equivalent strength, we can use another model previously applied successfully for iron protoporphyrin IX molecules adsorbed on a basal plane of a highly oriented pyrolytic graphite (HOPG) electrode. ${ }^{11}$ However, this model failed when analyzing the experimental p/s-ratio as a function of $\theta$ for Cyt-c 
(a)

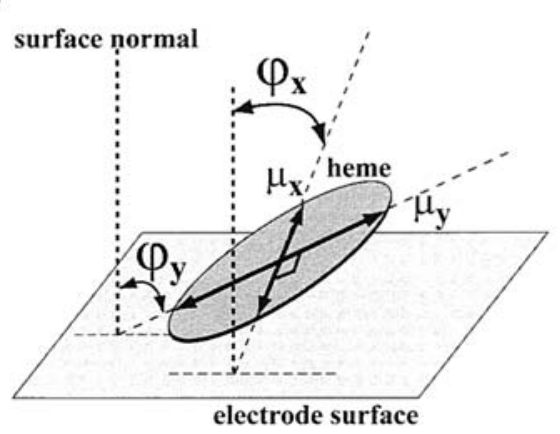

(b)

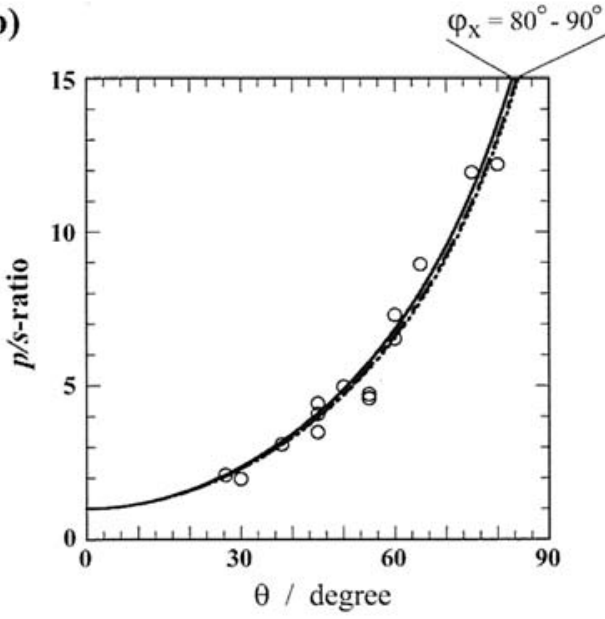

Fig. 3 (a) Schematic of the heme plane with two single dipoles responsible for Soret absorption, $\mu_{\mathrm{x}}$ and $\mu_{\mathrm{y}}$, on an electrode surface. The electric dipoles, $\mu_{\mathrm{x}}$ and $\mu_{\mathrm{y}}$, have, respectively, angles of $\varphi_{\mathrm{x}}$ and $\varphi_{\mathrm{y}}$ with respect to the normal of the electrode surface. (b) Plot of the p/s-ratio of ER signal at a wavelength $(\lambda)$ of $420 \mathrm{~nm}$ as a function of the incident angle, $\theta$, at a poly-Au electrode modified with a MUASAM + Cyt-c. Broken lines: working curves of plots of the p/s-ratio of ER signal vs. $\theta$. The experimental data are shown by open circles. In the calculation to obtain working curves, $\varphi_{\mathrm{y}}=10^{\circ}$ was presumed, and $\varphi_{\mathrm{x}}=80^{\circ}, 85^{\circ}$, and $90^{\circ}$ were used. Reproduced with permission from Ref. 12. Copyright 2006 American Chemical Society.

molecules on the SAM. What is meant by this fact is the need to separately calculate the interactions of two non-equivalent orthogonal dipoles with the electric field vectors of the light to describe the ER signal. Using the model shown in Fig. 3(a), we can simultaneously deal with two single dipoles $\left(\mu_{\mathrm{x}}\right.$ and $\left.\mu_{\mathrm{y}}\right)$, where their orientation angles $\left(\varphi_{\mathrm{x}}\right.$ and $\left.\varphi_{\mathrm{y}}\right)$ have a specific geometric relationship. ${ }^{12}$ The lines in Fig. 3(b) are working curves on the basis of this new model with an assumption that one of the dipole is upright to the electrode surface and the other is almost parallel to the surface. This corresponds to an almost vertical orientation of the heme plane to the electrode surface. The experimental $\mathrm{p} / \mathrm{s}-$ ratios well fit to the lines. If we intentionally tilted the heme obliquely, or rotated with respect to the main axis of the porphyrin ring in the model, the fitting always became worse. These results clearly point to the highly oriented hemes of Cyt-c molecules on the SAM-covered electrode. In light of the electrochemical kinetic data, ${ }^{18}$ the results support the model that the heme crevice of immobilized Cyt-c molecules is really facing the SAM surface, facilitating electron transfer with the underlying Au electrode.

2.1.3 PMTA spectroscopy as exemplified by Au-NP on ITO In PMTA spectroscopy, if we take the ac and dc transmitted
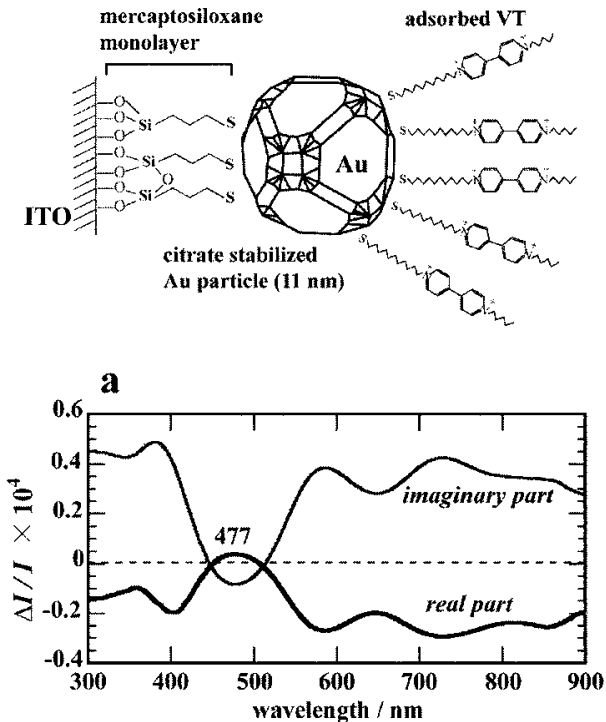

b

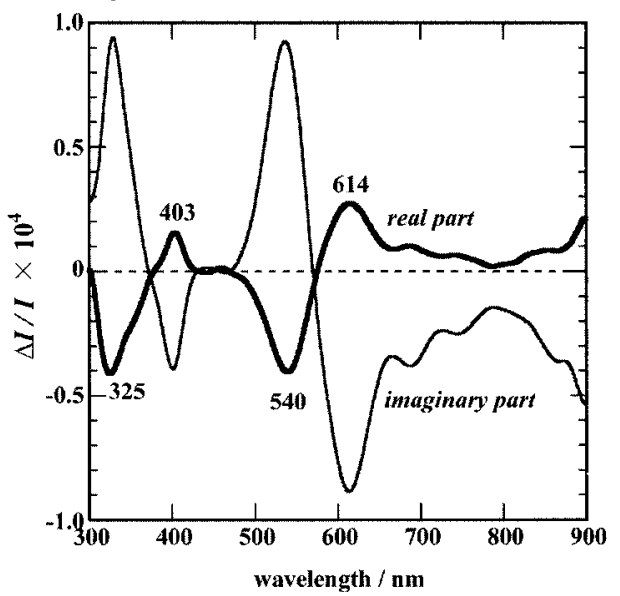

Fig. 4 Upper scheme: Structure of modified Au electrode with 3(triethoxysilyl)-1-propanethiol, citrate-stabilized Au-NP (11 nm), and VT. Lower figures: PMTA spectra for the electrode shown in upper scheme at $E_{\mathrm{dc}}=0.0 \mathrm{~V}\left(v s . \mathrm{Ag} / \mathrm{AgCl} / \mathrm{sat}^{\prime} \mathrm{KCl}\right)$ (a) and $E_{\mathrm{dc}}=E^{0^{\prime}}=$ $-0.452 \mathrm{~V}$ (b) at $f=14 \mathrm{~Hz}$ and $\Delta E_{\mathrm{ac}}=70 \mathrm{mV}_{\text {rms }}$. Reproduced with permission from Ref. 19. Copyright 2002 American Chemical Society.

light intensity instead of $I_{0} R_{\mathrm{ac}}$ and $I_{0} R_{\mathrm{dc}}$ in the ER method, respectively, the principle of the ac measurement of the transmission-absorption of the light is the same as ER spectroscopy. Typical PMTA spectra for an ITO electrode with immobilized Au-NPs together with irreversibly adsorbed VT molecules on the NPs are shown in Fig. $4 .{ }^{19}$ The PMTA signal, represented by $\Delta I / I$, signifies the absorbance at a more negative potential from which that at a more positive potential is subtracted.

Figure 4(a) shows the PMTA spectrum at $E_{\mathrm{dc}}$, where no redox reaction takes place. A positive-going peak of the real part around $477 \mathrm{~nm}$ in Fig. 4(a) is interpreted to be due to a blue shift of plasmon absorption band of Au-NP while changing the potential to more negative. ${ }^{19,20}$ The charging-discharging of AuNPs by electron transfer between ITO and immobilized Au-NP is the origin of the PMTA signal. When an Au-NP is more negatively charged, the plasmon band of Au-NP is shifted to shorter wavelength along with a sharpening and enhancement of the peak. ${ }^{19,20}$

When $E_{\mathrm{dc}}$ is set at the formal potential $\left(E^{0^{\prime}}\right)$ of the redox 
reaction of viologen, one may expect that the sum of the absorption spectrum of the viologen radical cation and the spectrum of Fig. 4(a) should be obtained, bearing in mind that the oxidized form of viologen is colorless. Although the occurrence of the viologen redox reaction was confirmed at $E^{0^{\prime}}$ by separate voltammetric measurements, what was obtained is the PMTA spectrum of Fig. 4(b) being far different from the above-mentioned prediction. A similar abnormality was also observed in the ER spectra at a Au electrode. ${ }^{19}$ It is likely that a strong interaction between viologen cation radical moieties and $\mathrm{Au}-\mathrm{NPs}$ under plasmon excitation of the NPs is the origin, because a change from the solution absorption spectrum of the reduced form of viologen occurred only around the plasmon band. This can not originate from an enhancement of the electromagnetic wave of the light in close proximity of the nanoparticle, but presumably from a charge-transfer like resonance. Further studies to examine the generality of this phenomenon using other dyes and to model the NP-dye interaction under plasmon excitation are required.

\subsection{Potential-modulated fluorescence spectroscopy at liquid/liquid interfaces}

Spectroelectrochemical approaches based on the linear potential sweep technique while the monitoring an optical signal, such as fluorescence and absorption, have been developed to study the ion-transfer reaction at liquid/liquid interfaces. $^{21-24} \quad$ By analyzing the appropriate optical signal instead of the overall current, the interfacial behavior of the target dye species could be studied in the absence of the interference with non-chromophoric species. A lock-in detection of ac-modulated optical signals under the potentialmodulation superimposed on a linear potential sweep improves the sensitivity and the selectivity to the interfacial phenomena of dye species. Potential-modulation spectroscopy, therefore, has been applied for the charge-transfer reaction across the liquid/liquid interface in terms of the potential-modulated reflectance $(\mathrm{PMR})^{25,26}$ and the potential-modulated fluorescence (PMF). ${ }^{27,28}$ In particular, the high sensitivity of PMF spectroscopy to interfacial phenomena has enabled us to use various fluorescent species, such as porphyrin, ${ }^{27-29}$ xanthene, ${ }^{30-32}$ and pyrene derivatives. ${ }^{33}$

The fundamental expression of PMF responses is derived within the framework of simple phenomenological models for ion-transfer and adsorption at a polarized liquid/liquid interface. $^{28}$ The sinusoidal perturbations of the Galvani potential difference across the interface $\left(\Delta_{0}^{\mathrm{w}} \phi \equiv \phi^{\mathrm{w}}-\phi^{\mathrm{o}}\right)$ is given in analogy with Eq. (1) as

$$
\Delta_{\circ}^{\mathrm{w}} \phi=\Delta_{\circ}^{\mathrm{w}} \phi_{\mathrm{dc}}+\Delta_{\circ}^{\mathrm{w}} \phi_{\mathrm{ac}} \exp (j \omega t)
$$

where $\Delta_{\circ}^{\mathrm{w}} \phi_{\mathrm{ac}}$ is the amplitude of the ac potential-modulation. The positive change of the Galvani potential difference would be a driving force for the transfer of cationic species from the aqueous to organic phases. The reverse-transfer process has been developed for anionic species.

Considering the fluorescent ion initially placed in the aqueous phase, the frequency-dependent fluorescence response $\left(\Delta F_{\mathrm{t}}\right)$ associated with a quasi-reversible ion transfer can be directly correlated with the faradaic ac current $\left(i_{\mathrm{f}, \mathrm{ac}}\right) .{ }^{21}$ In the case of the total internal reflection (TIR) condition (angle of incidence, $\theta$ ), $\Delta F_{\mathrm{t}}$ is described as ${ }^{27}$

$$
\Delta F_{\mathrm{t}}=\frac{4.606 \varepsilon \Phi I_{0}}{j \omega z F \cos \theta} i_{\mathrm{f}, \mathrm{ac}}
$$
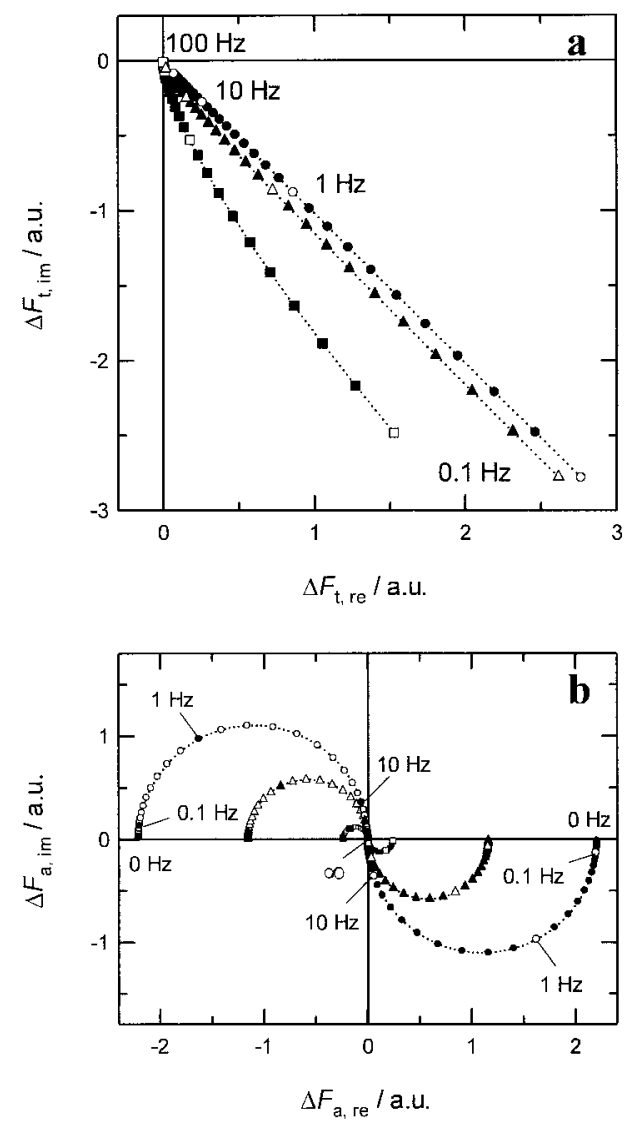

Fig. 5 Numerical simulations of the complex plane of PMF for (a) a quasi-reversible transfer and (b) an adsorption processes of mono cationic species. (a) The circle, triangle and square symbols relate to $k_{\mathrm{t}}=0.1,0.01$ and $0.001 \mathrm{~cm} \mathrm{~s}^{-1}$, respectively. $D^{\mathrm{W}}$ was taken as $5 \times$ $10^{-6} \mathrm{~cm}^{2} \mathrm{~s}^{-1}$. (b) The closed and open symbols depict the adsorption from the aqueous and organic sides of the interface. The circle, triangle and square relate to $k_{\mathrm{a}, \mathrm{dc}}=1 \times 10^{8}, 5 \times 10^{7}$ and $1 \times 10^{7} \mathrm{dm}^{3}$ $\mathrm{mol}^{-1} \mathrm{~s}^{-1}$, respectively. $k_{\mathrm{d}, \mathrm{dc}}$ was fixed to $10 \mathrm{~s}^{-1}$. Reproduced with permission from Ref. 28. Copyright 2001 American Chemical Society.

where $\varepsilon, \Phi, z, F$, and $S$, respectively, are the molar absorption coefficient, the fluorescence quantum yield, charge number of transferring ions, Faraday constant, and interfacial area. Numerical simulations of the PMF response for cationic species with various ion-transfer rate constants are illustrated in Fig. 5(a). In the case of cationic species, the positive change of the Galvani potential difference would be a driving force for iontransfer from the aqueous to organic phases. A PMF complex plane plot for cationic species appears in the forth quadrant. The real and imaginary components have almost the same magnitude for ion-transfer rate constants greater than $0.1 \mathrm{~cm} \mathrm{~s}^{-1}$. It should be noted that the second quadrant complex plane is applied for the transfer of anionic species, since the potential dependence of the direction of the transfer process is opposite. ${ }^{29,31}$

In the case that the adsorption of ionic species is described by the potential-dependent adsorption model based on the Langmuir isotherm, the frequency-dependent fluorescence response $\left(\Delta F_{\mathrm{a}}\right)$ associated with the adsorption of the fluorescent ion from the aqueous phase to the interface can be expressed as a function of the ac surface coverage $\left(\theta_{\mathrm{ac}}\right){ }^{28}$

$$
\Delta F_{\mathrm{a}}=2.303 \varepsilon \Phi I_{0} \Gamma_{\mathrm{s}} S \theta_{\mathrm{ac}},
$$




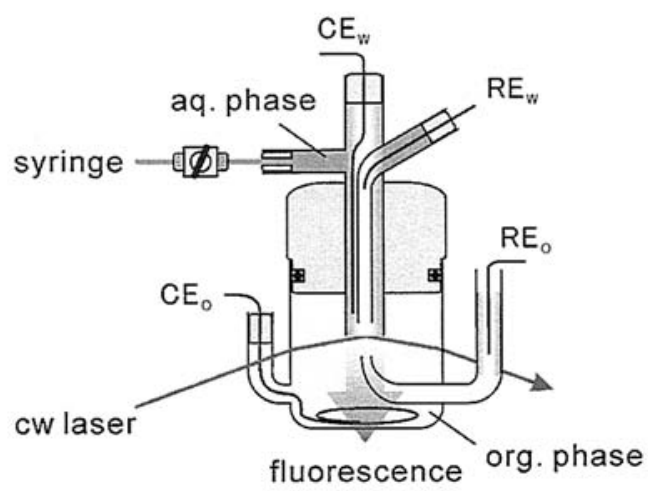

Fig. 6 Scheme of the setup for the PMF measurement. RE and CE are the reference and counter electrodes. Subscripts $w$ and o refer to the aqueous and organic phases, respectively.

where $\Gamma_{\mathrm{s}}$ is the saturated interfacial concentration. $\theta_{\mathrm{ac}}$ is described as

$$
\theta_{\mathrm{ac}}=\frac{b z F \Delta_{o}^{\mathrm{w}} \phi_{\mathrm{ac}}}{R T}\left[\frac{k_{\mathrm{a}, \mathrm{dc}} \alpha c_{\mathrm{dc}}\left(1-\theta_{\mathrm{dc}}\right)-k_{\mathrm{d}, \mathrm{dc}}(\alpha-1) \theta_{\mathrm{dc}}}{k_{\mathrm{a}, \mathrm{dc}} c_{\mathrm{dc}}+k_{\mathrm{d}, \mathrm{dc}}+j \omega}\right],
$$

where $b$ is the portion of the applied potential employed for adsorption process $\left(b^{\mathrm{w}}+b^{\text {org }} \leq 1\right), \alpha$ is the overall transfer coefficient for the adsorption process, $c_{\mathrm{dc}}$ is the bulk concentration, and $\theta_{\mathrm{dc}}$ is the dc surface coverage; $k_{\mathrm{a}, \mathrm{dc}}$ and $k_{\mathrm{d}, \mathrm{dc}}$ are the $\mathrm{dc}$ components of the adsorption and desorption rate constants at given potentials, respectively. The appropriate reverse sign is applied to Eq. (6) for the adsorption process from the organic phase because of an opposite potential dependence of the surface concentration. The complex plane of PMF for an adsorption process is described as a semicircle, as shown in Fig. 5(b), in contrast to that for a quasi-reversible transfer process, expressed as distorted lines approaching to the point of origin (cf. Fig. 5(a)). In addition, adsorption processes from aqueous and organic phases are clearly distinguishable due to the $180^{\circ}$ phase shift. For a cationic species, the complex plane of the adsorption process from the aqueous and organic phases appears in the fourth and second quadrants.

As shown in Figs. 5(a) and 5(b), the frequency response of the PMF signal gives a diagnostic criterion to clearly distinguish which interfacial process takes place (i.e., transfer or adsorption) and the location of the adsorption process (i.e., from aqueous or organic phase to the interface). A PMF complex plane plot measured around the formal transfer potential often shows an intermediate frequency response behavior involving both transfer and adsorption processes. ${ }^{28}$ The PMF spectroscopy can effectively extract the interfacial optical signal, especially for adsorption, from the fluorescence signal arising throughout the optical path in the bulk organic phase, even though the transfer response relatively decreases in the case of the fluorescent ion initially placed in the organic phase.

The typical experimental setup for the PMF measurement is schematically shown in Fig. 6. A glass tube filled with the aqueous phase is located into the organic phase, 1,2dichloroethane (DCE), and the water/DCE interface is polarized by a four-electrode potentiostat. The interface is illuminated under a TIR condition by a cw laser. The fluorescence signal is collected perpendicularly to the interface by a photomultiplier tube (PMT) and analyzed by a digital lock-in amplifier. Further details of the experimental setup have been reported previously. ${ }^{27,29}$

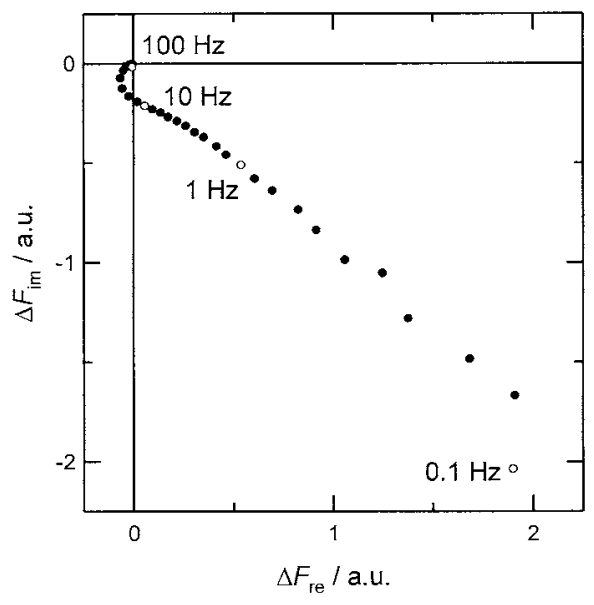

Fig. 7 Complex representation of the PMF responses for the transfer of $\mathrm{Ru}(\mathrm{bpy})_{3}{ }^{2+}$. The potential modulation at the formal transfer potential $(-0.10 \mathrm{~V})$ was varied from 0.1 to $100 \mathrm{~Hz}$ with an amplitude of $25 \mathrm{mV}_{\text {rms }}$. The concentration of $\mathrm{Ru}(\mathrm{bpy})_{3}{ }^{2+}$ was $2.5 \times$ $10^{-5} \mathrm{~mol} \mathrm{dm}^{-3}$. The excitation light source was ca. $20 \mathrm{~mW}$ at $442 \mathrm{~nm}$. Reproduced with permission from Ref. 28. Copyright 2001 American Chemical Society.

The combination of PMF spectroscopy and conventional electrochemical techniques has allowed us to uncover the interfacial adsorption and transfer processes of the fluorescent ions. For instance, tris $\left(2,2^{\prime}\right.$-bipyridyl)ruthenium(II) $\left(\mathrm{Ru}(\mathrm{bpy})_{3}{ }^{2+}\right)$ across the water/DCE interface showed quasi-reversible ion transfer features..$^{27}$ The frequency-dependent PMF response at the formal transfer potential is displayed in Fig. 7. At lower frequencies, the PMF complex plane plot exhibited a roughly linear relationship, in agreement with a numerical simulation in the absence of the adsorption process (Fig. 5(a)). The magnitudes of the real and imaginary components decrease with increasing the potential modulation frequency up to $c a .10 \mathrm{~Hz}$ in the fourth quadrant. The distorted feature at higher frequencies in the third quadrant is most likely to be due to attenuation of the amplitude of ac potential-modulation introduced by the interfacial capacitance and the uncompensated solution resistance. ${ }^{28}$

The PMF technique has also been applied to study the transfer reaction involving the adsorption process. The cationic zinc(II) porphyrin, $\quad 5,10,15,20$-tetrakis $(N$-methylpyridyl)porphyrinato zinc(II) $\left(\mathrm{ZnTMPyP}^{4+}\right)$, initially placed in the aqueous phase, exhibited a complex interfacial behavior, in which ZnTMPyP ${ }^{4+}$ was adsorbed at either side of the water/DCE interface. The corresponding PMF responses appeared in different quadrants of the complex plane, depending on whether the process took place from the aqueous or organic phase. In agreement with the theoretical model (Fig. 5(b)), the PMF responses for ZnTMPyP ${ }^{4+}$ measured at the potential corresponding to the adsorption process from the aqueous phase to the interface showed a distorted semicircle in the fourth quadrant (Fig. 8). By analyzing the frequency dependence of PMF, the Gibbs free energy of adsorption could be evaluated for porphyrins from the kinetic parameters, adsorption and desorption rate constants, as values between -30 and $-40 \mathrm{~kJ} \mathrm{~mol}^{-1} .^{28,29}$

The PMF response brings about an even deeper understanding of the emission properties of the fluorescent ion located in the interfacial region. The wavelength dependence of the PMF intensity (PMF spectrum), in principle, can be considered as the emission spectrum of the interfacial species without the contribution of dc signals from the bulk phase. The PMF spectrum for the cationic free base porphyrin, $\mathrm{H}_{2} \mathrm{TMPyP}^{4+}$, 


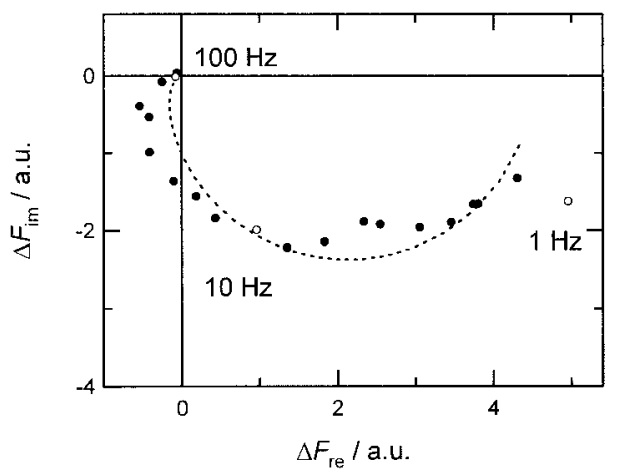

Fig. 8 Complex representation of the PMF responses for the adsorption of $\mathrm{ZnTMPyP}^{4+}$ at $-0.10 \mathrm{~V}$. The concentration of $\mathrm{ZnTMPyP}^{4+}$ was $2.5 \times 10^{-5} \mathrm{~mol} \mathrm{dm}{ }^{-3}$. The amplitude of potential modulation was $25 \mathrm{mV}_{\text {rms. }}$ The excitation light source was $c a .20$ $\mathrm{mW}$ at $442 \mathrm{~nm}$. The dashed line was obtained by curve-fitting with theoretical equations. Reproduced with permission from Ref. 28. Copyright 2001 American Chemical Society.

could be measured at a potential where the adsorption process takes place at the organic side of the interface (Fig. 9). ${ }^{29}$ The PMF spectrum indicates significant differences from the emission spectra measured in the bulk of the aqueous and organic phases. The wavelength of the maximum response in the PMF spectrum was found close to that of the emission spectrum measured in DCE. The peak ratio of the first and second maxima is, however, apparently different from that in the organic phase. This fact suggests that the solvation structure of interfacial species involving a long-range interaction correlated with the interfacial polarity is modified from both bulk species, and that it is adsorbed with the intermediate solvation structure. The dependence of the PMF intensity on the angle of the linear polarization of the excitation beam can also provide information about the molecular orientation of interfacial species. ${ }^{27,32}$ In the case of the adsorption of $\mathrm{ZnTMPyP}^{4+}$ at a water/DCE interface, for instance, the plane of the porphyrin ring is oriented roughly parallel to the interface. ${ }^{27}$ PMF spectroscopy allows us to elucidate the ion transfer and adsorption mechanisms along with a direct spectroscopic characterization of the interfacial species.

\section{Concluding Remarks and Future Scopes}

The practical features of potential-modulation spectroscopies were demonstrated by using a few examples taken from our recent studies for solid/liquid and liquid/liquid interfaces. In the application of protein modified electrodes to biosensors and bioelectrocatalytic systems, clarification of the state of immobilized protein is indispensable. The speculated models hitherto accumulated by a number of voltammetric works can be proved or denied clearly by ER and PMTA techniques through rather quick and easy measurements. These techniques are also applicable to track the behaviors of nano-materials at electrified interfaces. At liquid/liquid interfaces, highly sensitive PMF techniques can disclose which processes actually undergo there, yet fluorescent molecules should be used.

In response to increasing demand for the elucidation of molecular-level dynamics, although the methods described here can gain access to the averaged information over the illuminated surface area in regards to molecular structures and behavior, a wide range of applicability of the potential-modulation

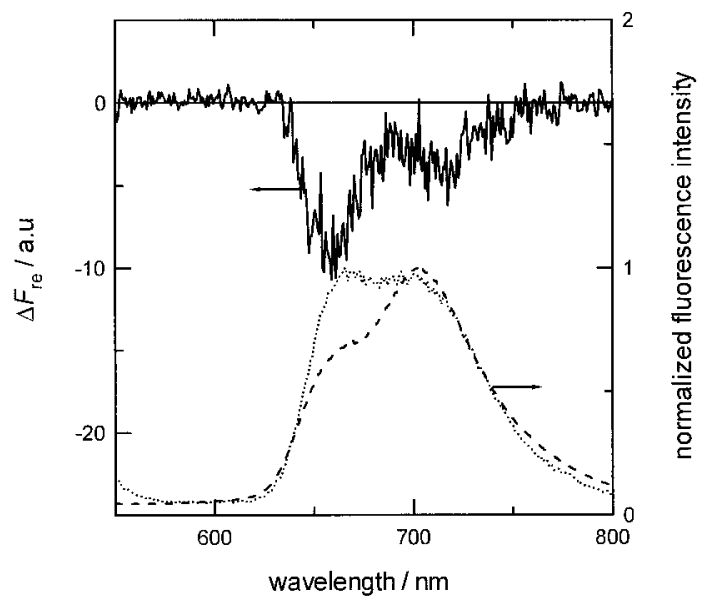

Fig. 9 Typical PMF spectrum for $\mathrm{H}_{2} \mathrm{TMPyP}^{4+}$ at the water/DCE interface. The PMF spectrum was measured as the real component of $\mathrm{PMF}$ at $0.04 \mathrm{~V}$. The potential modulation was $50 \mathrm{mV}_{\text {rms }}$ at $3 \mathrm{~Hz}$. The concentration of $\mathrm{H}_{2} \mathrm{TMPyP}^{4+}$ was $1.0 \times 10^{-4} \mathrm{~mol} \mathrm{dm}^{-3}$. The dashed and dotted lines refer to the emission spectra in the aqueous and organic phases, respectively. The excitation light source was a cw laser diode of $22.1 \mathrm{~mW}$ at $410 \mathrm{~nm}$. Reproduced with permission from Ref. 29. Copyright 2006 Elsevier.

technique is fascinating. Especially, the combined use of modulation optical signals with surface-selective or molecular imaging tools may find abundant opportunities to provide the basis of an accurate means to control the electrified interfaces. Coupling with theoretical developments in future, potentialmodulation spectroelectrochemical methods should become further sophisticated in order for the dynamics in double-layer region and key factors of the interfacial reactivity to be more deeply understood.

\section{Acknowledgements}

We gratefully acknowledge financial supports by Grant-in-Aids for Young Scientists (B) (No. 17750070 to H. N.) and Scientific Research (B) (No. 16350077 to T. S.) from the Ministry of Education, Culture, Sports, Science and Technology of Japan (MEXT).

\section{References}

1. "Spectroelectrochemistry: Theory and Practice", ed. R. J. Gale, 1988, Plenum Press, New York.

2. Y. Y. Tong, E. Oldfield, and A. Wieckowski in "Encyclopedia of Electrochemistry", ed. A. J. Bard, M. Stratmann, and E. J. Calvo, 2003, Vol. 2, Wiley-VCH, Weinheim, 147.

3. I. Burgess, V. Zamlynny, G. Szymanski, A. L. Schwan, R. J. Faragher, J. Lipkowski, J. Majewski, and S. Satija, J. Electroanal. Chem., 2003, 550, 187.

4. M. Shikata, Rev. Polarogr., 1954, 4, 15.

5. A. Prostak and W. N. Hansen, Phys. Rev., 1967, 160, 600.

6. J. D. E. McIntyre in "Optical Techniques in Electrochemistry”, ed. R. H. Müller, 1973, Vol. 9, Chap. 2, John Wiley and Sons, New York.

7. A. Bewick, K. Kunimatsu, B. S. Pons, and J. W. Russell, J. Electroanal. Chem., 1984, 160, 47. 
8. S. H. Kim and D. A. Scherson, Anal. Chem., 1992, 64, 3091.

9. S. Nakabayashi and A. Kira, J. Phys. Chem., 1991, 95, 9961.

10. T. Sagara in "Advances in Electrochemical Science and Engineering", ed. C. Alkire, D. M. Kolb, J. Lipkowski, and P. N. Ross, 2006, Vol. X, Wiley-VCH, Weinheim, 47.

11. T. Sagara, M. Fukuda, and N. Nakashima, J. Phys. Chem. $B, \mathbf{1 9 9 8}, 102,521$

12. T. Sagara, Y. Kubo, and K. Hiraishi, J. Phys. Chem. B, 2006, 110, 16550.

13. T. Sagara, H. Murase, M. Komatsu, and N. Nakashima, Appl. Spectrosc., 2000, 54, 316.

14. P. L. Edmiston, J. E. Lee, S. S. Cheng, and S. S. Saavedra, J. Am. Chem. Soc., 1997, 119, 560.

15. J. Zhou, J. Zheng, and S. Y. Jiang, J. Phys. Chem. B, 2004, 108, 17418.

16. K. Ataka and J. Heberle, J. Am. Chem. Soc., 2004, 126, 9445.

17. T. Sagara, N. Kaba, M. Komatsu, M. Uchida, and N. Nakashima, Electrochim. Acta, 1998, 43, 2183.

18. S. Imabayashi, T. Mita, and T. Kakiuchi, Langmuir, 2005, 21, 2474.

19. T. Sagara, N. Kato, A. Toyota, and N. Nakashima, Langmuir, 2002, 18, 6995.

20. A. Toyota, N. Nakashima, and T. Sagara, J. Electroanal.
Chem., 2004, 565, 335.

21. T. Kakiuchi and Y. Takasu, Anal. Chem., 1994, 66, 1853.

22. T. Kakiuchi and Y. Takasu, J. Electroanal. Chem., 1995, $381,5$.

23. T. Kakiuchi and Y. Takasu, J. Phys. Chem. B, 1997, 101, 5963.

24. Z. F. Ding, R. G. Wellington, P. F. Brevet, and H. H. Girault, J. Electroanal. Chem., 1997, 420, 35.

25. D. J. Fermín, Z. Ding, P. F. Brevet, and H. H. Girault, J. Electroanal. Chem., 1998, 447, 125.

26. Z. F. Ding, D. J. Fermín, P. F. Brevet, and H. H. Girault, J. Electroanal. Chem., 1998, 458, 139.

27. H. Nagatani, R. A. Iglesias, D. J. Fermín, P. F. Brevet, and H. H. Girault, J. Phys. Chem. B, 2000, 104, 6869.

28. H. Nagatani, D. J. Fermín, and H. H. Girault, J. Phys. Chem. B, 2001, 105, 9463.

29. H. Nagatani, T. Ozeki, and T. Osakai, J. Electroanal. Chem., 2006, 588, 99.

30. N. Nishi, K. Izawa, M. Yamamoto, and T. Kakiuchi, $J$. Phys. Chem. B, 2001, 105, 8162.

31. H. Nagatani, S. Suzuki, D. J. Fermín, H. H. Girault, and K. Nakatani, Anal. Bioanal. Chem., 2006, 386, 633.

32. T. Osakai, H. Yamada, H. Nagatani, and T. Sagara, J. Phys. Chem. C, 2007, 111, 9480.

33. K. Nakatani, H. Nagatani, D. J. Fermín, and H. H. Girault, J. Electroanal. Chem., 2002, 518, 1. 